\title{
Nucleocytoplasmic Gynodioecy
}

\author{
R. Doroteo ${ }^{1}$, J. A. Vargas $^{2}$ \\ ${ }^{1}$ Departamento de Ciencias Básicas, Instituto Tecnológico de Oaxaca, Oaxaca, México \\ ${ }^{2}$ CIIDIR-Oaxaca, Instituto Politécnico Nacional, Xoxocotlán, México \\ Email: rubendoroteo@prodigy.net.mx, jvargasm@ipn.mx
}

Received October 2, 2013; revised November 2, 2013; accepted November 9, 2013

Copyright (C) 2013 R. Doroteo, J. A. Vargas. This is an open access article distributed under the Creative Commons Attribution License, which permits unrestricted use, distribution, and reproduction in any medium, provided the original work is properly cited.

\begin{abstract}
We study the evolution of a gynodioecious species under mixed-mating through a nucleocytoplasmic male sterility model. We consider two cytoplasmic types and a nuclear locus with two alleles. Here, the interaction between one cytoplasmic type and a recessive nuclear male-sterility factor gives rise to only one female genotype, while the remaining types correspond to hermaphroditic plants. We include two fitness paramaters: the advantageous female fitness $t$ of females relative to that of hermaphrodites; and a silent and dominant cost of restoration, that is, a diminished fitness for plants carrying a dominant restorer gene relative to that of hermaphrodites. The parameter related to the cost of restoration is assumed to be present on outcrossing male fertility only. We find that every population converges to a stable population. We also determine the nature of the attracting stable population, which could be a nucleocytoplasmic polymorphism, a nuclear polymorphism or another population with some genotypes absent. This depends on the position of $t$ with respect to critical values expressed in terms of the other parameters and also on the initial population.
\end{abstract}

Keywords: Population Genetics; Gynodioecy; Dynamical System; Nucleocytoplasmic Interaction; Matrix of Linear Forms

\section{Introduction}

Gynodioecy is a reproductive system which occurs mainly in plants, e.g. ribwort plantain, Plantago lanceolata. It consists of the simultaneous presence of both hermaphroditic and female types. Such a reproductive system originates when a male-sterility mutation appears and spreads in a hermaphroditic population.

Early authors studied different kinds of interactions between cytoplasmic and nuclear male-sterility factors [1-3]. They formulated models which implied the elimination of one cytoplasm at equilibrium. More recent works [4-6] include the viability of equilibria with all types present even for pure outcrossing models.

In Section 2, we construct a model which considers two cytoplasmic types: $N$ and $S$; and a nuclear locus with two alleles: $M$ and $m$. Here, the interaction between the cytoplasmic type $S$ and the recessive nuclear male-sterility factor $m$ gives rise to only one female genotype $S m m$, while the remaining types ( $N M M, N M m$, $\mathrm{Nmm}, \mathrm{SMM}$ and $\mathrm{SMm}$ ) correspond to hermaphroditic plants. Our methods are algebraic, involving elementary commutative and computational algebra, as well as elementary algebraic geometry. We interpret our model as a rational map of the projective space $\mathbb{P}^{5}$ (see [7]). All symbolic computations were performed using Macaulay2 [8].

Our model incorporates mixed-mating, implying the use of a parameter for the selfing rate $s$, defined as the fraction of the progeny derived from selfing. Following Gouyon et al. [6], we include two fitness parameters: the advantageous female fitness $t$ of females relative to that of hermaphrodites; and a silent and dominant cost of restoration, expressed by the diminished fitness $w$ of plants carrying a dominant restorer gene relative to that of hermaphrodites. The works [5] and [9] consider similar models, but they include several additional fitness parameters. [5] and [6] are restricted to pure outcrossing reproduction. In [6], we find a biological discussion justifying the appearence of the parameter $w$.

The parameter $w$ is assumed to be present on male fertility for the genotypes $N M M$ and $N M m$; and only for outcrossing. In this way, we adapt a biological hypothesis to our model, to the effect that there is no pollen discounting, that is, the amount of pollen used for selfing is negligible in comparison with the total amount produced by the hermaphrodites. This hypothesis appears in 
Ross and Weir [10], cf. Holsinger et al. [11] and in Vargas and del Castillo [12] in a different form. It is partially supported by the observed positive association between outcrossing rates and pollen-ovule ratio found in a sample of different species of plants (see Cruden [13] and Damgaard and Abbott [14]).

Although our use of algebraic methods is relatively elementary, it allows us to construct models with total rigor, following logical, easy to verify steps, as in the present case.

An important part of any dynamical system is its geometry; in particular, the biological relevant geometry. In Section 3, we define these concepts precisely and determine this geometry rigorously, using the computational software mentioned above.

We obtain that a stable polymorphism exists, with all genotypes present, for suitable values of the three parameters. We call this population the "nucleocytoplasmic polymorphism", because it is unique for fixed parameter values; and we find an explicit expression for each genotype frequency.

There is another stable population, where the cytoplasm $N$ is absent, with the remaining three genotypes present. We say that this population is the "nuclear polymorphism". It also occurs for suitable values of the parameters and is unique for those values. We also find an expression for each genotype frequency.

In Section 4, we use the rich geometric information from the previous section to determine the critical values for the parameter $t$ in terms of the other parameters, corresponding to all dynamical bifurcations, including the appearance of the previous polymorphisms.

We find that every population converges to a stable population. The nature of the attracting stable population depends on the position of $t$ with respect to the critical values found before; and sometimes also on the initial population. We do not find limit cycles. The simulations that support some of our results are focused on the geometric data.

Section 5 is dedicated to the pure outcrossing case. Here, we can determine the complete geometry for the model. We also prove that the nuclear equilibria points are asymptotically stable. This material provides partial support for the general results.

\section{Formulation of the Model}

Consider one nuclear locus with two alleles $M$ and $m$; and suppose that there are also two cytoplasmic types: $N$ and $S$. This gives a total of six nucleocytoplasmic types: $N M M, N M m, N m m, S M M, S M m, S m m$. These are all assumed to be hermaphrodites, with the only one exception of $\mathrm{Smm}$, which is female, i.e. male sterile.

We work in projective space $\mathbb{P}^{5}$, a space whose points are the one dimensional subspaces from a six dimensional vector space $V$. Here, any point $x \in \mathbb{P}^{5}$ has as projective coordinates $\left(x_{0}, x_{1}, \cdots, x_{5}\right)$ the coordinates of any generator of its corresponding one dimensional subspace in $V$.

Let $\mathbb{P}_{+}^{5}$ be the subset of $\mathbb{P}^{5}$ consisting of all points which admit real homogeneous coordinates of the same sign, which we assume to be positive. We say that a point $x \in \mathbb{P}^{5}$ has biological relevance whenever $x \in \mathbb{P}_{+}^{5}$. We identify a given population $\mathcal{A}$ with the point $x=\left(x_{0}, \cdots, x_{5}\right) \in \mathbb{P}_{+}^{5}$ if each number $x_{i}$ is the frequency in $\mathcal{A}$ of the corresponding genotype:

$$
\begin{aligned}
& x_{0} \leftrightarrow N M M, x_{1} \leftrightarrow N M m, x_{2} \leftrightarrow N m m, \\
& x_{3} \leftrightarrow S M M, x_{4} \leftrightarrow S M m, x_{5} \leftrightarrow S m m .
\end{aligned}
$$

We will refer to the $i$-th genotype using this correspondence.

Conversely, any point $x=\left(x_{0}, \cdots, x_{5}\right) \in \mathbb{P}_{+}^{5}$ may be interpreted as a population, where each $x_{i}$ is the frequency of a genotype. This is achieved by choosing homogeneous coordinates such that $x_{0}+\cdots+x_{5}=1$.

It is necessary to consider separately the male and female contributions towards the next generation for each genotype. We construct the following matrices: $A_{i}$, describing the male contribution of the $i$-th genotype; and $B_{i}$, for its female contribution. Thus, the $j$-th row of $A_{i}$ gives the structure of a population obtained from outcrossing the male contribution of the $i$-th genotype with the female contribution of the $j$-th genotype. In this situation, the cytoplasmic alleles are transmitted through the females only.

$$
A_{0}=\left(\begin{array}{cccccc}
1 & 0 & 0 & 0 & 0 & 0 \\
\frac{1}{2} & \frac{1}{2} & 0 & 0 & 0 & 0 \\
0 & 1 & 0 & 0 & 0 & 0 \\
0 & 0 & 0 & 1 & 0 & 0 \\
0 & 0 & 0 & \frac{1}{2} & \frac{1}{2} & 0 \\
0 & 0 & 0 & 0 & 1 & 0
\end{array}\right), B_{0}=\left(\begin{array}{cccccc}
1 & 0 & 0 & 0 & 0 & 0 \\
\frac{1}{2} & \frac{1}{2} & 0 & 0 & 0 & 0 \\
0 & 1 & 0 & 0 & 0 & 0 \\
1 & 0 & 0 & 0 & 0 & 0 \\
\frac{1}{2} & \frac{1}{2} & 0 & 0 & 0 & 0 \\
0 & 1 & 0 & 0 & 0 & 0
\end{array}\right),
$$




$$
\begin{aligned}
& A_{1}=\left(\begin{array}{cccccc}
\frac{1}{2} & \frac{1}{2} & 0 & 0 & 0 & 0 \\
\frac{1}{4} & \frac{1}{2} & \frac{1}{4} & 0 & 0 & 0 \\
0 & \frac{1}{2} & \frac{1}{2} & 0 & 0 & 0 \\
0 & 0 & 0 & \frac{1}{2} & \frac{1}{2} & 0 \\
0 & 0 & 0 & \frac{1}{4} & \frac{1}{2} & \frac{1}{4} \\
0 & 0 & 0 & 0 & \frac{1}{2} & \frac{1}{2}
\end{array}\right), B_{1}=\left(\begin{array}{cccccc}
\frac{1}{2} & \frac{1}{2} & 0 & 0 & 0 & 0 \\
\frac{1}{4} & \frac{1}{2} & \frac{1}{4} & 0 & 0 & 0 \\
0 & \frac{1}{2} & \frac{1}{2} & 0 & 0 & 0 \\
\frac{1}{2} & \frac{1}{2} & 0 & 0 & 0 & 0 \\
\frac{1}{4} & \frac{1}{2} & \frac{1}{4} & 0 & 0 & 0 \\
0 & \frac{1}{2} & \frac{1}{2} & 0 & 0 & 0
\end{array}\right), \\
& A_{2}=\left(\begin{array}{cccccc}
0 & 1 & 0 & 0 & 0 & 0 \\
0 & \frac{1}{2} & \frac{1}{2} & 0 & 0 & 0 \\
0 & 0 & 1 & 0 & 0 & 0 \\
0 & 0 & 0 & 0 & 1 & 0 \\
0 & 0 & 0 & 0 & \frac{1}{2} & \frac{1}{2} \\
0 & 0 & 0 & 0 & 0 & 1
\end{array}\right), B_{2}=\left(\begin{array}{cccccc}
0 & 1 & 0 & 0 & 0 & 0 \\
0 & \frac{1}{2} & \frac{1}{2} & 0 & 0 & 0 \\
0 & 0 & 1 & 0 & 0 & 0 \\
0 & 1 & 0 & 0 & 0 & 0 \\
0 & \frac{1}{2} & \frac{1}{2} & 0 & 0 & 0 \\
0 & 0 & 1 & 0 & 0 & 0
\end{array}\right), \\
& A_{3}=\left(\begin{array}{cccccc}
1 & 0 & 0 & 0 & 0 & 0 \\
\frac{1}{2} & \frac{1}{2} & 0 & 0 & 0 & 0 \\
0 & 1 & 0 & 0 & 0 & 0 \\
0 & 0 & 0 & 1 & 0 & 0 \\
0 & 0 & 0 & \frac{1}{2} & \frac{1}{2} & 0 \\
0 & 0 & 0 & 0 & 1 & 0
\end{array}\right), B_{3}=\left(\begin{array}{cccccc}
0 & 0 & 0 & 1 & 0 & 0 \\
0 & 0 & 0 & \frac{1}{2} & \frac{1}{2} & 0 \\
0 & 0 & 0 & 0 & 1 & 0 \\
0 & 0 & 0 & 1 & 0 & 0 \\
0 & 0 & 0 & \frac{1}{2} & \frac{1}{2} & 0 \\
0 & 0 & 0 & 0 & 1 & 0
\end{array}\right), \\
& A_{4}=\left(\begin{array}{cccccc}
\frac{1}{2} & \frac{1}{2} & 0 & 0 & 0 & 0 \\
\frac{1}{4} & \frac{1}{2} & \frac{1}{4} & 0 & 0 & 0 \\
0 & \frac{1}{2} & \frac{1}{2} & 0 & 0 & 0 \\
0 & 0 & 0 & \frac{1}{2} & \frac{1}{2} & 0 \\
0 & 0 & 0 & \frac{1}{4} & \frac{1}{2} & \frac{1}{4} \\
0 & 0 & 0 & 0 & \frac{1}{2} & \frac{1}{2}
\end{array}\right), B_{4}=\left(\begin{array}{cccccc}
0 & 0 & 0 & \frac{1}{2} & \frac{1}{2} & 0 \\
0 & 0 & 0 & \frac{1}{4} & \frac{1}{2} & \frac{1}{4} \\
0 & 0 & 0 & 0 & \frac{1}{2} & \frac{1}{2} \\
0 & 0 & 0 & \frac{1}{2} & \frac{1}{2} & 0 \\
0 & 0 & 0 & \frac{1}{4} & \frac{1}{2} & \frac{1}{4} \\
0 & 0 & 0 & 0 & \frac{1}{2} & \frac{1}{2}
\end{array}\right),
\end{aligned}
$$




$$
A_{5}=\left(\begin{array}{cccccc}
0 & 1 & 0 & 0 & 0 & 0 \\
0 & \frac{1}{2} & \frac{1}{2} & 0 & 0 & 0 \\
0 & 0 & 1 & 0 & 0 & 0 \\
0 & 0 & 0 & 0 & 1 & 0 \\
0 & 0 & 0 & 0 & \frac{1}{2} & \frac{1}{2} \\
0 & 0 & 0 & 0 & 0 & 1
\end{array}\right), B_{5}=\left(\begin{array}{cccccc}
0 & 0 & 0 & 0 & 1 & 0 \\
0 & 0 & 0 & 0 & \frac{1}{2} & \frac{1}{2} \\
0 & 0 & 0 & 0 & 0 & 1 \\
0 & 0 & 0 & 0 & 1 & 0 \\
0 & 0 & 0 & 0 & \frac{1}{2} & \frac{1}{2} \\
0 & 0 & 0 & 0 & 0 & 1
\end{array}\right) .
$$

To compensate for its male sterility, the genotype Smm has associated an enhanced female fertility, so that

$$
t=\frac{\text { female fertility of } \mathrm{Smm}}{\text { female fertility of hermaphrodites }} \geq 1 .
$$

We assume that selection acts against plants that carry a dominant restorer gene $M$ together with the cytoplasm $N$. Thus, the genotypes $N M M$ and $N M m$ have associated a diminished male fertility, so that

$$
w=\frac{\text { outcrossing male fertility of } N M M, N M m}{\text { outcrossing male fertility of hermaphrodites }}<1 .
$$

The parameter $t$ is introduced through the female fitness matrix $F$, while the male sterility and $w$ are introduced through the male fitness matrix $G$.

$$
F=\left(\begin{array}{llllll}
1 & 0 & 0 & 0 & 0 & 0 \\
0 & 1 & 0 & 0 & 0 & 0 \\
0 & 0 & 1 & 0 & 0 & 0 \\
0 & 0 & 0 & 1 & 0 & 0 \\
0 & 0 & 0 & 0 & 1 & 0 \\
0 & 0 & 0 & 0 & 0 & t
\end{array}\right), G=\left(\begin{array}{cccccc}
w & 0 & 0 & 0 & 0 & 0 \\
0 & w & 0 & 0 & 0 & 0 \\
0 & 0 & 1 & 0 & 0 & 0 \\
0 & 0 & 0 & 1 & 0 & 0 \\
0 & 0 & 0 & 0 & 1 & 0 \\
0 & 0 & 0 & 0 & 0 & 0
\end{array}\right) .
$$

The evolution of this reproductive system has to be formulated using all possible crosses between genotypes.

We first construct the outcrossing matrix $Q$ as follows:

$$
\begin{aligned}
& Q=12\left[x_{0}\left(w F A_{0}+G B_{0}\right)+x_{1}\left(w F A_{1}+G B_{1}\right)\right. \\
& +x_{2}\left(F A_{2}+G B_{2}\right)+x_{3}\left(F A_{3}+G B_{3}\right) \\
& \left.+x_{4}\left(F A_{4}+G B_{4}\right)+x_{5}\left(0 \cdot F A_{5}+t G B_{5}\right)\right] .
\end{aligned}
$$

We next construct the selfing matrix $S$, writing as its $i$-th row half the sum of the $i$-th row of the matrix $A_{i}$ with the $i$-th row of $B_{i}$, for $0 \leq i \leq 5$, except that the last row must be zero. Thus,

$$
S=\left(\begin{array}{cccccc}
1 & 0 & 0 & 0 & 0 & 0 \\
14 & 12 & 14 & 0 & 0 & 0 \\
0 & 0 & 1 & 0 & 0 & 0 \\
0 & 0 & 0 & 1 & 0 & 0 \\
0 & 0 & 0 & 14 & 12 & 14 \\
0 & 0 & 0 & 0 & 0 & 0
\end{array}\right)
$$

Finally, we obtain the mixed-mating matrix $T=s h S+(1-s) Q$, where $0 \leq s \leq 1$ is the selfing rate; and $h=x_{0}+\cdots+x_{5}$ is a homogenizing factor, first introduced by Holgate [15], it equals one in any biological context. The evolution map is $\varphi: \mathbb{P}^{5} \rightarrow \mathbb{P}^{5}$, given by

$$
\varphi(x)=x T=\left(y_{0}, \cdots, y_{5}\right),
$$

for $x \in \mathbb{P}^{5}$. It provides the genotype frequencies for the next generation of any population $x \in \mathbb{P}_{+}^{5}$.

\section{Geometry of the Dynamical System}

We call trajectory any sequence $\left\{z_{i} \mid i=0,1,2, \cdots\right\} \subset \mathbb{P}^{5}$ such that $z_{i+1}=\varphi\left(z_{i}\right)$, for all $i$. We start with the elementary observation that in case there is a trajectory $z_{0}, z_{1}, z_{2}, \cdots$ such that $\lim _{i \rightarrow \infty} z_{i}=w$, then the point $w$ must be a fixed point for the map, i.e. a point with $\varphi(w)=w$, or else it must be a fundamental point, i.e. a point where $\varphi(w)$ is not defined because all its homogeneous coordinates would vanish.

We say that the fixed point $v$ is stable whenever given a neighborhood $\mathcal{U}$ of $v$, in the classical topology, we can find a neighborhood $\mathcal{W} \subseteq \mathcal{U}$ of $v$ such that all points in $\mathcal{W}$ are sent to $\mathcal{U}$ by $\varphi^{n}$, for $n$ sufficiently large. We say that the fixed point $v$ is asymptotically stable, when, in addition to the above condition, there exists an open set $\mathcal{B}$, such that $\lim _{n \rightarrow \infty} \varphi^{n}(x)=v$ for all $x \in \mathcal{B}$. These definitions are adapted from those usually given for dynamical systems, see Hirsch and Smale [16].

We will determine the geometry of the fixed and fundamental points of biological interest. If $f_{1}, \cdots, f_{n}$ are homogeneous polynomials, we write their locus $Z\left(f_{1}, \cdots, f_{n}\right)=\left\{p \in \mathbb{P}^{5} \mid f_{1}(p)=\cdots=f_{n}(p)=0\right\} \quad ; \quad$ and similarly for infinite collections of homogeneous polynomials. If $\mathfrak{a}$ is a homogeneous ideal of the polynomial ring $\mathbb{R}\left[x_{0}, \cdots, x_{5}\right], Z(\mathfrak{a})$ is the locus of all of the homogeneous elements of $\mathfrak{a}$.

Proposition 1. All fixed and fundamental points of biological interest for the map $\varphi$ are among the following:

1) Those on the line $L=Z\left(x_{1}, x_{2}, x_{4}, x_{5}\right)$, which consists of fixed points, except for the fundamental point 
$f=(1,0,0, s w-s-w, 0,0) \notin \mathbb{P}_{+}^{5}$.

2) The fundamental point $a=(0,0,0,0,0,1)$.

3) The fixed point $b=(0,0,1,0,0,0)$.

4) The fixed point $c=(0,0, s, 0,0, t(1-s)-1)$, provided $t>\frac{1}{1-s}$.

5) The pair of fixed points associated to the ideal $W \subset R=\mathbb{C}[s, t, w]\left[x_{0}, \cdots, x_{5}\right]$ generated by the following five forms:

$$
\begin{aligned}
p_{1}= & (1-w) x_{0}-x_{3}, \\
p_{2}= & (1-w) x_{1}-x_{4}, \\
p_{3}= & (1-w) x_{2}-t x_{5}, \\
q_{1}= & t x_{0} x_{1}+t x_{1}^{2}+t x_{1} x_{2}-x_{0} x_{1}-4 x_{0} x_{2}-x_{1} x_{2}, \\
q_{2}= & t(s t-s w+s-t+1)\left(x_{0}+x_{1}\right) \\
& \quad+[t(s t-t+1)+s(1-w)] x_{2} .
\end{aligned}
$$

6) The pair of fixed points associated to the ideal $N \subset R$ generated by the following forms:

$$
\begin{aligned}
& x_{0}, x_{1}, x_{2}, \\
& (1-s) t\left(-x_{3}-x_{4}+x_{5}\right)+2 s x_{5}+2\left(x_{3}+x_{4}\right), \\
& t\left(x_{3} x_{4}-4 x_{3} x_{5}+x_{4}^{2}-x_{4} x_{5}\right)-2 x_{3} x_{4} .
\end{aligned}
$$

Proof 1. (1 - 4): The fixed and fundamental points of $\varphi$ are associated to the ideal I generated by the $2 \times 2$ minors from the $2 \times 6$ matrix whose rows are $x$ and $\varphi(x)$. It is easy to verify that the points $a, b$ and $c$ and the line $L$ satisfy I. It is equally easy to verify that among all these points, exactly the points $a$ and $f$ are fundamental.

(5 - 6): The ideal $I$ is difficult to handle. However, the ideals $W$ and $N$ are easier to manage and they contain substantial information about fixed points of biological interest. The ideal $\left(y_{0}, \cdots, y_{5}\right)$ of fundamental points, is also easy to handle and it does not produce any further points of biological relevance.

By Bézout's theorem applied to the plane $\mathbb{P}^{2}$ with homogeneous coordinates $x_{0}, x_{1}, x_{2}$, we obtain that $W$ describes a pair of points, which are the intersection of a line and a conic.

Similarly, we see that $N$ describes a pair of points, by Bézout's theorem applied to the plane with homogeneous coordinates $x_{3}, x_{4}, x_{5}$.

We calculate the transporter

$$
W: I=\{z \in R \mid z I \subseteq W\},
$$

obtaining as a result an ideal containing the nonzero parameter $t$. This means that the points corresponding to $W$ are fixed or fundamental points for our map. Then we saturate $W$ with the ideal generated by $y_{0}, \cdots, y_{5}$, the coordinates of $\varphi$. The result is $W$. Thus, the points described by $W$ are fixed points of $\varphi$.

An analogous process proves that the points described by $N$ are also fixed points.

Finally, we saturate the ideal $I$ with the ideal of fundamental points; then we saturate the result successively with $W, N$ and the ideals for the line $L$, the points $a, b$ and $c$. The result from the above process is an ideal whose primary decomposition shows no points of biological relevance. $\square$

The fixed points in

$$
\mathcal{P}=\left\{x=\left(x_{0}, \cdots, x_{5}\right) \in \mathbb{P}_{+}^{5} \mid x_{0} \cdots x_{5} \neq 0\right\}
$$

correspond to polymorphisms with all genotypes present.

Corollary 1. All fixed points in $\mathcal{P}$ for the map $\varphi$ are among those associated to the ideal $W$.

Our immediate problem is to determine the nature of the pairs of points associated to $W$ and $N$. First, we fix the notation. Let

$$
\begin{aligned}
& \alpha=\frac{1+2 s(1-w)}{1-s w}, \\
& \beta_{1,2}=\frac{1 \pm \sqrt{1+4 s(1-s)(1-w)}}{2(1-s)}, \\
& \gamma=\frac{1+s(1-w)}{1-s}, \\
& \delta_{1,2}=\frac{2+s(1-w) \pm 2 \sqrt{1+2 s-s^{2}-2 s w+s^{2} w}}{4-s(5-w)} .
\end{aligned}
$$

Lemma 1. These quantities satisfy:

- $0<\alpha<\beta_{1}<\gamma<\delta_{1}$, where the last inequality requires $s(5-w)<4$.

- $1 /(1-s)<\beta_{1}<\gamma<2 /(1-s)$.

- $\delta_{1}<\delta_{2}$ if and only if $s(5-w)<4$.

- $\beta_{2}, \delta_{2}<0$.

Proof 2. We prove only the first assertion. The remaining assertions admit analogous proofs.

The inequality $0<\alpha$ is clear. Since both numerators and denominators involved are positive, we verify that $\alpha<\beta_{1}$ as follows: Since

$$
\begin{aligned}
2 & (1-s w)\left[(1-s)\left(\beta_{1}+\alpha\right)-1\right] \\
= & (\sqrt{1+4 s(1-s)(1-w)}-1)(1-s w)+2(1-s) \\
& \cdot[1+2 s(1-w)], \\
4 & (1-s)(1-s w)^{2}\left(\beta_{1}-\alpha\right)\left[(1-s)\left(\beta_{1}+\alpha\right)-1\right] \\
= & (1-s w)^{2} 4 s(1-s)(1-w)+4(1-s)[1+2 s(1-w)] \\
& \cdot(1-s w)-4(1-s)^{2}[1+2 s(1-w)]^{2},
\end{aligned}
$$

we have 


$$
\begin{aligned}
& 4(1-s)(1-s w)^{2}\left(\beta_{1}-\alpha\right)\left[(1-s)\left(\beta_{1}+\alpha\right)-1\right] \\
& =(1-s w)^{2}[1+4 s(1-s)(1-w)] \\
& -\{2(1-s)[1+2 s(1-w)]-(1-s w)\}^{2} \\
& =4 s^{3}(1-s)(1-w)(2-w)^{2}>0 .
\end{aligned}
$$

Here, we observe that $1 /(1-s)<\beta_{1}<\beta_{1}+\alpha$. We verify that $\beta_{1}<\gamma$ as follows: Since

$$
2(1-s)\left(\gamma-\beta_{1}\right)=1+2 s(1-w)-\sqrt{1+4 s(1-s)(1-w)},
$$

it suffices to see that

$$
\begin{aligned}
& {[1+2 s(1-w)]^{2}-[1+4 s(1-s)(1-w)]} \\
& =4 s^{2}(2-w)(1-w)>0 .
\end{aligned}
$$

We verify that $\gamma<\delta_{1}$ provided $s(5-w)<4$ as follows: Since both numerators and denominators involved are positive and

$$
\begin{aligned}
& (1-s)[4-s(5-w)]\left(\delta_{1}-\gamma\right) \\
& =2(1-s) \sqrt{1+2 s-s^{2}-2 s w+s^{2} w}-\{[1+s(1-w)] \\
& \quad \cdot[4-s(5-w)]-(1-s)[2+s(1-w)]\},
\end{aligned}
$$

it suffices to see that

$$
\begin{aligned}
& 4(1-s)^{2}\left(1+2 s-s^{2}-2 s w+s^{2} w\right) \\
& -\{[1+s(1-w)][4-s(5-w)]-(1-s)[2+s(1-w)]\}^{2} \\
& =s^{3}[4-s(5-w)](1-w)(2-w)^{2}>0 .
\end{aligned}
$$

One fixed point $v$, described by $W$, has homogeneous coordinates

$$
\begin{aligned}
& v_{0}=-2 t(t-1)+s\left(-1-t^{2}(w-3)-2 t(w-1)+w\right)-\sqrt{B}, \\
& v_{1}=4 t(t-1)+s\left(-1+t^{2}(w-5)+2 t(w-1)+w\right)+\sqrt{B}, \\
& v_{2}=2 t[1+s(1-w)-t(1-s)], \\
& v_{3}=(1-w) v_{0}, v_{4}=(1-w) v_{1}, v_{5}=(1-w) t v_{2} .
\end{aligned}
$$

where

$$
\begin{aligned}
& B=s(t-1)(w-1) \times\left\{8 t\left(t^{2}-1\right)\right. \\
& \left.+s\left[1+t^{3}(w-9)+11 t(w-1)-w+t^{2}(5 w-13)\right]\right\} .
\end{aligned}
$$

One fixed point $u$, satisfying $N$, has homogeneous coordinates

$$
\begin{aligned}
& u_{0}=u_{1}=u_{2}=0, \\
& u_{3}=(t-1)[(1-s) t-s]-\sqrt{D}, \\
& u_{4}=-\left[(1-s) t^{2}-(s-s t+2 t)\right]+\sqrt{D}, \\
& u_{5}=(1-s) t-2 .
\end{aligned}
$$

where

$$
\begin{aligned}
D= & 2 t[t(1-s)+2 s][t(1-s)-2] \\
& +\left(t^{2}-2 t-s+s t-s t^{2}\right)^{2} .
\end{aligned}
$$

The point $u$ has biological relevance exactly when the inequality $(1-s) t>2$ holds. The other point satisfying $N$ never has biological meaning.

The ideal $N$ corresponds to a pair of fixed points, one of which describes the associated nuclear polymorphism, i.e. where exactly the genotypes SMM, SMm, Smm are present.

Proposition 2. We have $v \in \mathcal{P}$ if and only if $v \in \mathbb{P}_{+}^{5}$ if and only if $\beta_{1}<t<\gamma$.

Proof We first observe that

$$
\begin{aligned}
& {\left[-2 t(t-1)+s\left(-1-t^{2}(w-3)-2 t(w-1)+w\right)\right]^{2}-B} \\
& =(1-s w)(\alpha-t)\left(t-\beta_{2}\right)\left(\beta_{1}-t\right) .
\end{aligned}
$$

Hence, $v_{0}>0$ if and only if $t<\alpha$ or $t>\beta_{1}$.

We observe that $v_{1}=\left(t-\delta_{2}\right)\left(t-\delta_{1}\right)+\sqrt{B}$ and also $B-\left[\left(t-\delta_{2}\right)\left(t-\delta_{1}\right)\right]^{2}=(1-s)(t-\gamma)\left(t-\beta_{2}\right)\left(\beta_{1}-t\right)$.

Hence, $v_{1}>0$ if and only if $\beta_{1}<t<\gamma$.

We finally observe that $v_{2}=2 t(1-s)(\gamma-t)$. Hence $v_{2}>0$ for $t<\gamma$.

We have verified that $\beta_{1}<t<\gamma$ implies

$$
v_{0}>0, v_{1}>0, v_{2}>0 \text {. }
$$

This clearly also implies

$$
v_{3}>0, v_{4}>0, v_{5}>0 .
$$

Thus, $v \in \mathcal{P}$ if $\beta_{1}<t<\gamma$. The reciprocal also follows from the above calculations, as well as the remaining equivalence involving $\mathbb{P}_{+}^{5}$.

Multiple simulations, both algebraic and graphic strongly suggest that the second point satisfying $W$ never has biological meaning. Thus, we have nearly complete understanding of the biologically relevant geometry for our rational map $\varphi$. We state this information in biological terms:

Proposition 3. The populations in equilibrium for our gynodioecy model are the following:

1) The nucleocytoplasmic polymorphism corresponding to the point $v$ with coordinates given by Equations (7). This is possible if and only if the parameters satisfy

$$
\beta_{1}<t<\gamma .
$$

2) The nuclear polymorphism corresponding to the point $u$ with coordinates given by Equations (8). This is possible if and only if the parameters satisfy

$$
t>\frac{2}{1-s} \text {. }
$$

3) The populations without the nuclear allele $m$ 
corresponding to the line $L$ in Proposition 1 (1).

4) The population without the nuclear allele $M$, corresponding to the point $c$ in Proposition 1 (4). This is possible if and only if the parameters satisfy

$$
t>\frac{1}{1-s} \text {. }
$$

5) The population with unique genotype $\mathrm{Nmm}$, corresponding to the point $b$ in Proposition 1 (3).

\section{The Dynamics}

We observe that for a given population $x \in \mathbb{P}_{+}^{5}$, the expression $x_{0}+x_{1}+x_{2}$ gives the frequency of cytoplasm $N$, whereas $x_{3}+x_{4}+x_{5}$ gives the frequency of cytoplasm $S$, once we choose coordinates with $x_{0}+\cdots+x_{5}=1$. Let

$$
\lambda(x)=\frac{\text { frequency of cytoplasm } N}{\text { frequency of cytoplasm } S} .
$$

Our analysis, including many simulations, provides enough understanding for the biologically relevant dynamics of $\varphi$, which exhibits a complex behavior. We obtained that every biologically relevant point converges to a fixed point. Also, every fixed point is an attractor, i.e. every fixed point is asymptotically stable. If $p$ is an attractor, we define its domain of attraction $D(p)$ as the set $\left\{x \in \mathbb{P}^{5} \mid \lim _{n \rightarrow \infty} \varphi^{n}(x)=p\right\}$. We also define the biologically relevant domain of attraction:
$D_{+}(p)=D(p) \cap \mathbb{P}_{+}^{5}$.

Result 1. The rational map $\varphi$ has the following approximate behavior:

1) If $x \in \mathbb{P}_{+}^{5}, \quad t<\frac{2}{1-s}$ and $\lambda(x)<1$, then $\lim _{n \rightarrow \infty} \varphi^{n}(x) \in L$.

2) If $x \in \mathbb{P}_{+}^{5}, \quad 1<t<\frac{1}{1-s}$ and $\lambda(x)>1$, then $\lim _{n \rightarrow \infty} \varphi^{n}(x)=b$.

3) If $x \in \mathbb{P}_{+}^{5}, \frac{1}{1-s}<t<\beta_{1}$ and $\lambda(x)>1$, then $\lim _{n \rightarrow \infty} \varphi^{n}(x)=c$.

4) If $x \in \mathbb{P}_{+}^{5}, \quad \beta_{1}<t<\gamma$ and $\lambda(x)>1$, then $\lim _{n \rightarrow \infty} \varphi^{n}(x)=v$, where $v$ is given by Equations (7).

5) If $x \in \mathbb{P}_{+}^{5}, \quad \gamma<t<\frac{2}{1-s}$ and $\lambda(x)>1$, then $\lim _{n \rightarrow \infty} \varphi^{n}(x) \in L$, for all $x \in \mathbb{P}_{+}^{5}$.

6) If $x \in \mathbb{P}_{+}^{5}$ and $t>\frac{2}{1-S}$, then $\lim _{n \rightarrow \infty} \varphi^{n}(x)=u$, where $u$ is given by Equations (8).

Table 1 supports the assertions in Result 1. The column $x$ indicates the initial population. The column $y$ indicates the expected attractor fixed point according to the Result.

\begin{tabular}{|c|c|c|c|c|}
\hline$(s, t, w)$ & $x$ & $\lambda(x)$ & $y_{3000}$ & $y$ \\
\hline $1:(.9,1.2, .5)$ & $(1,5,3,3,1,4)$ & 1.12 & $(0,0,1,0,0,0)$ & $b=(0,0,1,0,0,0)$ \\
\hline $1:(.9,1.2, .5)$ & $(1,2,3,6,8,5)$ & 0.31 & $(.53,0,0, .46,0,0)$ & $y \in L$ \\
\hline $1:(.8,3.0, .4)$ & $(5,2,3,1,2,3)$ & 1.6 & $(0,0,1,0,0,0)$ & $b=(0,0,1,0,0,0)$ \\
\hline $1:(.8,3.0, .4)$ & $(1,2,3,6,5,3)$ & 0.42 & $(.49,0,0, .5,0,0)$ & $y \in L$ \\
\hline $2:(.7,3.4, .5)$ & $(1,2,1,4,3,2)$ & 0.4 & $(.34,0,0, .65,0,0)$ & $y \in L$ \\
\hline $2:(.6,2.7, .2)$ & $(6,4,1,4,3,2)$ & 1.2 & $(0,0, .88,0,0, .12)$ & $c=(0,0, .88,0,0, .12)$ \\
\hline $2:(.6,2.7, .2)$ & $(1,4,1,4,3,2)$ & 0.6 & $(.51,0,0, .48,0,0)$ & $y \in L$ \\
\hline $3:(.5,2.5, .24)$ & $(5,4,3,4,3,2)$ & 1.3 & $v$ & $v=(.12, .16, .38, .09, .12, .12)$ \\
\hline $3:(.5,2.5, .24)$ & $(1,4,3,5,3,2)$ & 0.8 & $(.42,0,0, .57,0,0)$ & $y \in L$ \\
\hline $3:(.4,2.0, .2)$ & $(6,4,3,5,3,2)$ & 1.3 & $v$ & $v=(.05, .12, .50, .04, .10, .20)$ \\
\hline $3:(.4,2.0, .2)$ & $(1,2,3,5,3,2)$ & 0.6 & $(.35,0,0, .64,0,0)$ & $y \in L$ \\
\hline $4:(.4,3.0, .2)$ & $(1,2,8,2,3,2)$ & 1.57 & $(.02,0,0, .98,0,0)$ & $y \in L$ \\
\hline $4:(.4,3.0, .2)$ & $(1,2,1,2,3,6)$ & 0.36 & $(.02,0,0, .98,0,0)$ & $y \in L$ \\
\hline $5:(.3,4.0, .4)$ & $(4,6,8,2,3,4)$ & 2 & $(0,0,0, .3, .5, .19)$ & $\mu=(0,0,0, .3, .5, .19)$ \\
\hline $5:(.3,4.0, .4)$ & $(2,3,1,4,3,4)$ & 0.54 & $(0,0,0, .3, .5, .19)$ & $\mu=(0,0,0, .3, .5, .19)$ \\
\hline $5:(.7,7.0, .4)$ & $(2,7,6,4,3,4)$ & 1.36 & $(0,0,0, .84, .13, .03)$ & $\mu=(0,0,0, .84, .13, .03)$ \\
\hline $5:(.7,7.0, .4)$ & $(2,1,6,4,8,5)$ & 0.52 & $(0,0,0, .84, .13, .03)$ & $\mu=(0,0,0, .84, .13, .03)$ \\
\hline
\end{tabular}

Table 1. Simulations supporting Result 1. 
Remarks. For easy reference, we restate Result 1 in more biological terms:

1) If $1<t<\frac{2}{1-s}$, every population with $\lambda(x)<1$, converges to an equilibrium population without the nuclear allele $m$.

2) If $1<t<\frac{1}{1-s}$, every population with $\lambda(x)>1$, converges to an equilibrium population with unique genotype $\mathrm{Nmm}$.

3) If $\frac{1}{1-s}<t<\beta_{1}$, every population with $\lambda(x)>1$, converges to an equilibrium population without the nuclear allele $M$.

4) If $\beta_{1}<t<\gamma$, every population with $\lambda(x)>1$, converges to the nucleocytoplasmic polymorphism.

5) If $\gamma<t<\frac{2}{1-s}$, every population with $\lambda(x)>1$, converges to an equilibrium population without the nuclear allele $m$.

6) If $t>\frac{2}{1-s}$, every population converges to the nuclear polymorphism.

Remarks. The equation $\lambda(x)=1$ being linear, defines a hyperplane in $\mathbb{P}^{5}$. Regarding Result 1 , it is convenient to consider the half-spaces

$H_{1}=\left\{x \in \mathbb{P}^{5} \mid \lambda(x)>1\right\}$ and its opposite

$H_{2}=\left\{x \in \mathbb{P}^{5} \mid \lambda(x)<1\right\}$. In terms of domains of attraction, this Result reads as follows:

1) If $1<t<\gamma$, then $\bigcup_{z \in L} D_{+}(z)$ nearly equals $H_{2} \cap \mathbb{P}_{+}^{5}$.

2) If $\frac{1}{1-s}<t<\beta_{1}$, then $D_{+}(b)$ nearly equals $H_{1} \cap \mathbb{P}_{+}^{5}$.

3) If $\frac{1}{1-s}<t<\beta_{1}$, then $D_{+}(c)$ nearly equals $H_{1} \cap \mathbb{P}_{+}^{5}$.

4) If $\beta_{1}<t<\gamma$, then $D_{+}(v)$ nearly equals $H_{1} \cap \mathbb{P}_{+}^{5}$.

5) If $\gamma<t<\frac{2}{1-s}$, then $\bigcup_{z \in L} D_{+}(z)$ nearly equals $\mathbb{P}_{+}^{5}$.

6) If $t>\frac{2}{1-s}$, then $D_{+}(u)$ nearly equals $\mathbb{P}_{+}^{5}$.

Result 2. We list several refinements to Result 1.

1) For $1<t<\frac{1}{1-s}$, we have:

a) $D_{+}(b)$ stretches towards a region around the line segment $\overrightarrow{a b}$ consisting of points $x \in \mathbb{P}_{+}^{5}$ such that $\lambda(x)<1$.

b) $\bigcup_{z \in L} D_{+}(z)$ stretches towards a region around the line $L$ consisting of points $x \in \mathbb{P}_{+}^{5}$ such that $\lambda(x)>1$.

2) For $\frac{1}{1-s}<t<\beta_{1}$, we have:

a) $\bigcup_{z \in L} D_{+}(z)$ stretches towards a region around the line $L$ consisting of points $x \in \mathbb{P}_{+}^{5}$ such that $\lambda(x)>1$.

b) Here, $\lambda(c)>1$ always holds. However, for values of the parameters such that $\lambda(c)$ is close to one, $D_{+}(c)$ stretches to a small region around $c$ consisting of points $x \in \mathbb{P}_{+}^{5}$ such that $\lambda(x)<1$.

3) For $\beta_{1}<t<\gamma$, we have:

a) $\bigcup_{z \in L} D_{+}(z)$ stretches towards a region around the line $L$ consisting of points $x \in \mathbb{P}_{+}^{5}$ such that $\lambda(x)>1$.

b) $\bigcup_{z \in L} D_{+}(z)$ stretches towards a region around the point $b$ consisting of points $x \in \mathbb{P}_{+}^{5}$ such that $\lambda(x)>1$.

c) $\bigcup_{z \in L} D_{+}(z)$ stretches towards a region around the point $c$ consisting of points $x \in \mathbb{P}_{+}^{5}$ such that $\lambda(x)>1$.

d) Depending on the values of the parameters, $D_{+}(v)$ stretches towards a region around the line segment $a v$ consisting of points $x \in \mathbb{P}_{+}^{5}$ such that $\lambda(x)<1$.

Table 2 supports the assertions in Result 2.

\section{The Case of Pure Outcrossing}

We obtain a model for this case setting the parameter $s=0$.

\subsection{Geometry}

Here, the set of fundamental points of the map $\varphi$ is an algebraic set $K$. It is the union of two linear spaces of dimensions three and one, respectively:

- $Z\left(w x_{1}+2 x_{2}+x_{4}, w x_{0}-x_{2}+x_{3}\right)$

- $Z\left(x_{3}+\frac{1}{2} x_{4}, x_{1}+2 x_{2}, x_{0}-x_{2}, t x_{5}+\frac{1}{2} x_{4}\right)$

The point

$$
a=(0,0,0,0,0,1) \in Z\left(w x_{1}+2 x_{2}+x_{4}, w x_{0}-x_{2}+x_{3}\right)
$$

is the unique element of $K$ with biological relevance.

The set of fixed points of $\varphi$ has closure equal to an algebraic set $J$ which admits different descriptions according to the values of the parameter $t$ :

1) $t=1$. Here, $J$ has four irreducible components: $C_{1}$, a conic; $C_{2}$ a curve of degree 3 ; and two lines $L_{1}$ and $L_{2}$. The curve $C_{2}$ contains no points of biological relevance because its points satisfy the equations

$$
x_{0}+x_{1}+x_{2}=0, x_{3}+x_{4}+x_{5}=0 .
$$


Table 2. Simulations supporting the refinements in Result 2.

\begin{tabular}{|c|c|c|c|c|}
\hline$(s, t, w)$ & $x$ & $\lambda(x)$ & $y 3000$ & $y$ \\
\hline $1 f:(.9,1.2,5)$ & $(1,1,6,1,1,8)$ & 0.8 & $(0,0,1,0,0,0)$ & $y \in L$ \\
\hline $1 f:(.8,3,4)$ & $(.1,2,2, .3, .4,6)$ & 0.34 & $(0,0,1,0,0,0)$ & $y \in L$ \\
\hline $1 g:(.8,3,4)$ & $(5, .1,1,4, .1, .1)$ & 1.24 & $(.58,0,0, .42,0,0) \in L$ & $b$ \\
\hline $1 g:(.7,3,3)$ & $(6, .1, .1,5, .1, .1)$ & 1.19 & $(.57,0,0, .43,0,0) \in L$ & $b$ \\
\hline $2 a:(.7,3.4,3)$ & $(5, .1,1,4, .1, .1)$ & 1.24 & $(.59,0,0, .41,0,0) \in L$ & $c$ \\
\hline $2 b:(.9,10.2, .3)$ & $(0,0, .98, .5, .5, .02)$ & 0.96 & $c=(0,0, .98,0,0, .02)$ & $y \in L$ \\
\hline $3 a:(.9,12, .3)$ & $(6, .1,1,5, .1, .1)$ & 1.19 & $(.56,0,0, .44,0,0) \in L$ & $v$ \\
\hline $3 b:(.9,16,3)$ & $(.1,1,2, .1, .1, .1)$ & 7.3 & $(.14,0,0, .86,0,0) \in L$ & $v$ \\
\hline $3 c:(.9,16,3)$ & $(.1, .1, .9, .1, .1, .6)$ & 1.375 & $(.15,0,0, .85,0,0) \in L$ & $v$ \\
\hline $3 d:(.9,13, .3)$ & $(.27, .05,043, .19, .036, .8)$ & 0.73 & $v=(.27, .05, .43, .19, .036, .023)$ & $y \in L$ \\
\hline
\end{tabular}

The remaining components are these:

$$
\begin{aligned}
C_{1}= & Z\left(x_{4}^{2}-4 x_{3} x_{5}, x_{5}-(1-w) x_{2},\right. \\
& \left.x_{4}-(1-w) x_{1}, x_{3}-(1-w) x_{0}\right), \\
L_{1}= & Z\left(x_{0}, x_{1}, x_{3}, x_{4}\right), L_{2}=Z\left(x_{1}, x_{2}, x_{4}, x_{5}\right) .
\end{aligned}
$$

2) $t \neq 1,2$. In the general case, $J$ has six irreducible components: Five points plus the line $L_{2}$ from the previous case. Three of the five points never have biological meaning. The remaining points are:

$$
\begin{aligned}
& b=(0,0,1,0,0,0), \\
& u=\left(0,0,0, \frac{t^{2}-t}{t-2}-\sqrt{\frac{t^{3}}{t-2}}, \sqrt{\frac{t^{3}}{t-2}}-t, 1\right) .
\end{aligned}
$$

The point $u$ has biological meaning exactly in case $t>2$.

3) $t=2$. Here, the situation is as above, but instead of the point $u$ we have the point $u^{\prime}=(0,0,0,1,0,0)$.

\subsection{Dynamics}

It is convenient to study the map $\tau: \mathbb{R}^{6} \rightarrow \mathbb{R}^{6}$ induced by $\varphi$ defined as

$$
\tau\left(x_{0}, \cdots, x_{5}\right)=\frac{1}{h}\left(y_{0}, \cdots, y_{5}\right),
$$

where the $y_{i}$ are any set of homogeneous coordinates for the map $\varphi$; and $h=y_{0}+\cdots+y_{5}$. This map sends the complement of $\{a\}$ in the convex set $\mathcal{C}$ of points with nonnegative coordinates in the hyperplane $x_{0}+\cdots+x_{5}=1$ into itself. Since this map is defined over the real numbers and does not involve projective space, it is suitable to carry out simulations; and it qualifies as a traditional model for the biological phenomena.

Theorem 1. The point

$$
u=\left(0,0,0, \frac{t^{2}-t}{t-2}-\sqrt{\frac{t^{3}}{t-2}}, \sqrt{\frac{t^{3}}{t-2}}-t, 1\right)
$$

is asymptotically stable if $t>2$.

Proof. We use the Lyapunov criterion, see [16], applied to the transformation $\tau$. Let $\mathcal{J}=\left.($ jacobian $\tau)\right|_{u}$. Then $\operatorname{det}(\lambda-\mathcal{J})=\lambda^{2}\left(\lambda-r_{1}\right)\left(\lambda-r_{2}\right)\left(\lambda-r_{3}\right)\left(\lambda-r_{4}\right)$, where

$$
r_{1}=\frac{t-2}{2 t(t-1)}, r_{2}=2 r_{1}, r_{3,4}=\frac{1}{4 t^{2}(t-1)^{2}} \times\left\{a+b \sqrt{\frac{t^{3}}{t-2}} \pm \sqrt{k^{2}-8 t^{2}(t-1)^{2}\left(c+d \sqrt{\frac{t^{3}}{t-2}}\right)}\right\}
$$

Here,

$$
\begin{aligned}
& a=6 t^{2}-11 t^{3}+6 t^{4}-t^{5}, b=8-20 t+18 t^{2}-7 t^{3}+t^{4}, \\
& c=-8 t+20 t^{2}-18 t^{3}+7 t^{4}-t^{5}, \\
& d=16-32 t+24 t^{2}-8 t^{3}+t^{4}, \\
& k=-\left(a+b \sqrt{\frac{t^{3}}{t-2}}\right) .
\end{aligned}
$$

Using elementary calculus we obtain that $t>2$ implies $\left|r_{1}\right|,\left|r_{2}\right|,\left|r_{3}\right|,\left|r_{4}\right|<1$

Since $s=0$, we have $\alpha=\beta_{1}=\gamma=1$; consequently the interval for the polymorphism reduces to the point $t=1$. Here, the map $\varphi$ may be restricted to the plane

$$
\begin{gathered}
D=\left\{\left(x_{0}, \cdots, x_{5}\right) \in \mathbb{P}^{5} \mid x_{3}=(1-w) x_{0},\right. \\
\left.x_{4}=(1-w) x_{1}, x_{5}=(1-w) x_{2}\right\}
\end{gathered} .
$$


Proposition 4. The dynamical system defined by the iteration of $\psi=\left.\varphi\right|_{D}$ has the following properties:

1) The determinantal algebraic set of fixed and fundamental points of the map, given by the $2 \times 2$ minors from the $2 \times 6$ matrix with rows $x$ and $\psi(x)$ is $C_{1}$, the smooth conic above.

2) The set of fundamental points for $\psi$ is the single point

$$
B=(1,-2,1,(1-w),-2(1-w),(1-w)) \in C_{1} .
$$

3) The set of fixed points of $\psi$ is $C_{1} \backslash\{B\}$.

4) The tangent line to $C_{1}$ at $B$ is $L=Z(h) \cap D$, where $h=x_{0}+x_{1}+x_{2}$.

5) Here, $\psi(L \backslash\{B\})=B$.

6) Every point $x \in D \backslash L$ is sent to the intersection of $C_{1} \backslash\{B\}$ with the line $\overline{x B}$. The domain of attraction of $P \in C_{1} \backslash\{B\}$ is $\overline{P B} \backslash\{B\}$.

This result admits an easy proof; and it implies that the dynamical system reduces to a double Hardy-Weinberg system, with one step convergence to a stable population with polymorphism. It is only in case $t=1$ that stable polymorphisms appear.

Remarks. For the present pure outcrossing model, we have the following facts:

1) The stable nuclear gynodioecy remains for $t>2$.

2) For $1<t<2$ the surviving genotypes are $N M M$ and $S M M$, whereas only the genotype $S M M$ survives, if $t=2$. When $t$ is close to 2 , the genotype $S M m$ disappears very slowly.

3) If we delete the cost of restoration, which is achieved by the condition $w=1$; and $H \subset \mathbb{P}^{5}$ is the plane $x_{3}=x_{4}=x_{5}=0$, we have that $\left.\varphi\right|_{H}$ is the classical Hardy-Weinberg map. In this case, the points in the conic $C_{1}$ are attractors.

4) Consider the induced map $\eta: \mathbb{P}^{2} \rightarrow \mathbb{P}^{2}$, obtained from $\varphi$ and the projection $\pi: \mathbb{P}^{5} \rightarrow \mathbb{P}^{2}$ given by $\pi\left(x_{0}, \cdots, x_{5}\right)=\left(x_{3}, x_{4}, x_{5}\right)$, such that the following diagram commutes:

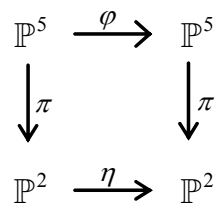

The rational map $\eta$ provides a nuclear gynodioecy model analogous to the androdioecy model studied in [12], instead of the gynodioecy model studied there. This happens because we have not presently assumed any "no pollen discounting" hypothesis for the cytoplasm $S$, which was present in [12].

\section{Discussion}

We consider that our contribution to the understanding of gynodioecy has two aspects: First of all, the rigor in the model formulation and in the determination of the underlying geometry. Secondly, the pertinence and necessity of our methods should be manifest. Unfortunately, these methods are not yet sufficiently developed to provide for a proof of the dynamical results. Nevertheless, we have exhibited a hierarchy for the different stable populations in equilibrium, in terms of biologically sound parameters.

In regard to the present gynodioecy model, our study is directed towards the general case with respect to mixed-mating, that is, for situations with selfing rate satisfying $0<s<1$. We found a precise interval $J=\left[\beta_{1}, \gamma\right]$ such that the inclusion of the female fitness $t \in J$ is a necessary and sufficient condition for the existence of a nucleocytoplasmic polymorphism. Table 3 shows values for the bounds of $J$. The table also shows that the interval size increases, as well as the allowed values of $t$, for larger values of selfing; and for smaller values of $w$, which reflect higher costs of restauration.

Our results remain valid and present no discontinuity for the pure outcrossing case $s=0$, where we find that $t=1$ is the only value for which there exists nucleocytoplasmic polymorphism. Thus, it is of a negligible nature, reinforcing the notion that this polymorphism requires self-fertilization. This result tends to contradict the assertion in [5] that this polymorphism "is attainable for a large set of parameter values", although their model differs from ours by their inclusion of an extra cytoplasmic fitness parameter.

The model in [6] is similar to our pure outcrossing case. Their assumptions differ from ours by their inclusion of two nuclear loci.

Although our model includes the pure selfing case $s=1$, our results do not apply to that case. We consider this situation of lesser mathematical and biological interest. We can see directly that the map $\varphi$ becomes linear in this case; and also that

Table 3. Bounds for female fitness associated to nucleocytoplasmic polymorphism in terms of selfing rate and cost of retoration,

\begin{tabular}{cccccccc}
\hline$s$ & $w$ & $\beta_{1}$ & $\gamma$ & $s$ & $w$ & $\beta_{1}$ & $\gamma$ \\
\hline 0.2 & 0.8 & 1.288796 & 1.30 & 0.2 & 0.6 & 1.325446 & 1.35 \\
0.2 & 0.4 & 1.360272 & 1.40 & 0.2 & 0.2 & 1.393521 & 1.45 \\
0.4 & 0.8 & 1.743156 & 1.80 & 0.4 & 0.6 & 1.813696 & 1.93 \\
0.4 & 0.4 & 1.879790 & 2.07 & 0.4 & 0.2 & 1.941385 & 2.20 \\
0.6 & 0.8 & 2.614734 & 2.80 & 0.6 & 0.6 & 2.720544 & 3.10 \\
0.6 & 0.4 & 2.819235 & 3.40 & 0.6 & 0.2 & 2.912077 & 3.70 \\
0.8 & 0.8 & 5.155184 & 5.80 & 0.8 & 0.6 & 5.301785 & 6.60 \\
0.8 & 0.4 & 5.441088 & 7.40 & 0.8 & 0.2 & 5.574085 & 8.20 \\
\hline
\end{tabular}




$$
\lim _{n \rightarrow \infty} \varphi^{n}(x)=\left(x_{0}+\frac{x_{1}}{2}, 0, x_{2}+\frac{x_{1}}{2}, x_{3}+\frac{x_{4}}{2}, 0,0\right),
$$

for any $x \in \mathbb{P}^{5}$. If we assume that the cost of restoration is present in selfing, we obtain

$$
\lim _{n \rightarrow \infty} \varphi^{n}(x)=\left(0,0, \frac{w x_{1}}{2(2-w)}+x_{2}, x_{3}+\frac{x_{4}}{2}, 0,0\right),
$$

for $0<w<1$.

\section{REFERENCES}

[1] E. Caspari, S. Watson and W. Smith, "The Influence of Cytoplasmic Pollen Sterility on Gene Exchange between Population," Genetics, Vol. 53, 1966, pp. 741-746. http://www.genetics.org/content/53/4/741.full.pdf

[2] D. Charlesworth and F.R. Ganders, "The Population Genetics of Gynodioecy with Cytoplasmic-Genic Male-Sterility," Heredity, Vol. 43, 1979, pp. 213-218. http://dx.doi.org/10.1038/hdy.1979.76

[3] G. S. Watson and E. Caspari, "The Behavior of Cytoplasmic Pollen Sterility in Populations," Evolution, Vol. 14, No. 1, 1960, pp. 56-63. http://dx.doi.org/10.2307/2405922

[4] M. F. Bailey, L. F. Delph and C. M. Lively, "Modelling Gynodioecy: Novel Scenarios for Maintaining Polymorphism," The American Naturalist, Vol. 161, No. 5, 2003, pp. 762-776. http://dx.doi.org/10.1086/374803

[5] M. Dufay, P. Touzet, S. Maurice and J. Cuguen, "Modelling the Maintenance of Male-Fertile Cytoplasm in a Gynodioecious Population," Heredity, Vol. 99, 2007, pp. 349-356. http://dx.doi.org/10.1038/sj.hdy.6801009

[6] P. H. Gouyon, F. Vichot and J. M. M. van Damme, "Nuclear-Cytoplasmic Male Sterility: Single-Point Equilibria versus Limit Cycles," The American Naturalist, Vol. 134, No. 4, 1991, pp. 498-513.

http://www.jstor.org/discover/10.2307/2462377?uid=373 8664uid=2129 uid=2uid=70uid $=4$ sid $=21102745687371$
[7] R. Hartshorne, "Algebraic Geometry," In: Graduate Texts in Mathematics, Vol. 52, Springer, New York, 1977.

[8] D. R. Grayson and M. E. Stillman, "Macaulay2, a Software System for Research in Algebraic Geometry." http://www.math.uiuc.edu/Macaulay2

[9] X. Delannay, P. H. Gouyon and G. Valdeyron, "Mathematical Study of the Evolution of Gynodioecy with Cytoplasmic Inheritance under the Effect of a Nuclear Restorer Gene," Genetics, Vol. 99, 1981, pp. 169-181. http://www.ncbi.nlm.nih.gov/pmc/articles/PMC1214488/

[10] M. D. Ross and B. S. Weir, "Maintenance of Male Sterility in Plant Populations III. Mixed Selfing and Random Mating," Heredity, Vol. 35, 1975, pp. 21-29. http://dx.doi.org/10.1038/hdy.1975.64

[11] K. E. Holsinger, M. W. Feldman and F. B. Christiansen, "The Evolution of Self-Fertilization in Plants," The American Naturalist, Vol. 124, 1984, pp. 446-453. http://www.jstor.org/discover/10.2307/2461472?uid=373 8664uid=2129uid $=2$ uid $=70$ uid $=4$ sid $=21102745754551$

[12] J. A. Vargas and R. F. del Castillo, "Nuclear Androdioecy and Gynodioecy," Journal of Mathematical Biology, Vol. 47, No. 3, 2003, pp. 199-221. http://dx.doi.org/10.1007/s00285-003-0200-3

[13] R. W. Cruden, "Pollen-Ovule Ratios: A Conservative Indicator of Breeding Systems in Flowering Plants," Evolution, Vol. 31, 1977, pp. 32-46.

http://www.jstor.org/discover/10.2307/2407542?uid=373 8664uid=2129uid=2 uid=70 uid=4 sid=21102745773421

[14] C. Damgaard and R. J. Abbott, "Positive Correlations between Selfing Rate and Pollen-Ovule Ratio within Plant Populations," Evolution, Vol. 49, 1995, pp. 214-217. http://www.jstor.org/discover/10.2307/2410307?uid=373 8664uid=2129uid=2 uid=70 uid=4 sid=21102745773421

[15] P. Holgate, "Selfing in Genetic Algebras," Journal of Mathematical Biology, Vol. 6, No. 2, 1978, pp. 197-206. http://dx.doi.org/10.1007/BF02450789

[16] M. Hirsch and S. Smale, "Differential Equations, Dynamical Systems and Linear Algebra," Academic Press, New York, 1974. 